de un gramme de trypsine commerciale fournit, après dilution et filtrations, plus de deux litres de liquide nutritif à un prix de revient très inférieur à celui des solutions peptonées couramment employees en bactériologie (1).

Ce milieu nous semble done intéressant à plus d'un titre. Ayant à le faire connaître, nous espérons avoir fourni aux chercheurs dont il retiendra l'attention assez de documents pour qu'ils puissent a pprécier sa valeur.

\title{
LA GRAISSE DU SANG ET LA GRAISSE DU LAIT PENDANT LA LACTATION
}

par

Ch. PORCHER

Docteur ès-Sciences physiques. et

Professeur de Nutrition des animaux

Université Cornell, Ithaca (N. Y.).

Depuis les recherches faites au laboratoire de l'Ecole Vétérinaire de Lyon, nous a vons à citer le travail de l'un de nous, L. A. MaynaRD, en collaboration a vec C. M. McCAY, et enfin, une étude toute récente de J. MARCQ, déjà cités l'un et l'autre.

1 «Depuis quelques années, dit J. Marce, on s'efforce, dans plusieurs instituts zootechniques, de mettre au point des données certaines sur la composition chimique et biologique du sang en relation avec la constitution et la productivité des animaux. Les recherches ont porté sur le dosage de la substance sèche du sang, sa teneur en hémoglobine, le nombre de globules rouges et blanes, $\mathrm{y}$ compris les variétés de ces derniers, l'alcalinité du sang, les phénomènes d'isoagglutination, etc. Il faut bien reconnaître que, jusqu'à présent tout au moins, on n'a pu dégager de conclusions vraiment applicables à la pratique. Il s'agit d'ailleurs d'épreuves délicates dont les résultats peuvent être au surplus influencés par de multiples facteurs ambiants."

" Il est pourtant raisonnable de persévérer dans cette voie. On aura rendu un grand service à l'éleveur le jour où l'on pourra lui dire : "Parmi les jeunes taureaux d'élevage que vous possédez, celui-ci est certainement le plus intéressant quant à ses possibilités beurrières. "Semblable raisonnement peut être applicable aux jeunes femelles. De ce point de vue, nous avons pensé que le dosage de la matière grasse du sang, du "taux butyreux sanguin ", suivant une

(1) A Poligny, le litre de ce liquide nous revient actuellement à moins de deux francs, tandis qu'un liquide peptoné à $2 \%$ nous coûte 7 francs le litre environ: 
expression heureuse de $\mathrm{Ch}$. Porcher, constituerait peut-être un indice sérieux d'un bon génotype. Au préalable, il importait de procéder à l'examen du sang de femelles laitières en comparaison a vec la richesse en matière grasse du lait fourni par elles. En effet, en Belgique tout au moins, il est difficile de trouver des taureaux reproducteurs à descendance actuellement bien connue. Il faudra attendre quelques années avant de pouvoir tirer des conclusions adéquates. Notre attention est attirée de ce côté. »

"Toutefois en vertu de nos connaissances en matière d'hérédité, il est vraisemblable que des corrélations existant entre le taux butyreux sanguin de la femelle et la richesse de son lait ont toutes chances d'exister entre le taux butyreux sanguin du mâle et son pouvoir de transmission de l'aptitude beurrière à sa descendance. Bref, nous soumettons l'idée aux chercheurs et nous ferons connaître dès maintenant les données essentielles que nous possédons, avec l'espoir que nos résultats futurs seront confirmés ou infirmés par d'autres travaux similaires. "

MARCQ a opéré sur deux vaches âgées de 4 ans ayant accouché à un mois de distance seulement, donnant un rendement en lait sensiblement égal avec une richesse nettement différente en matière grasse. Les prélèvements du sang dans la jugulaire furent faits à la même heure. Le dosage de la matière grasse du sang fut effectué suivant la méthode Röse-Gottlieb légèrement modifiée par HUYGE. Cette méthode ne donnerait peut-être pas des résultats aussi probants que la méthode Kumagawa modifiée par Lameland et par TERroine et ses élèves et qui est celle que nous avons utilisée dans nos recherches. Néanmoins, les résultats obtenus sont plus qu'intéressants, mais très encourageants :

Date des essais

17-4-29

$1-5-29$

29-5-29

(après la mise en pâture)

18-6-29

10-8-29
Vache $n^{\circ}$ I

Sang : 0,307

Lait : 5,3

Sang : 0,310

Lait : 4,5

Sang : 0,240

Lait : 4,1

Sang : 0,280

Lait : 4,00

Sang : 0,320

Lait : 3,3
Vache $n^{\circ}$ II

Sang : 0,298

Lait : 3,5

Sang : 0,298

Lait : 3,6

Sang : 0,220

Lait : 2,5

Sang : 0,270

Lait : 3,2

Sang : 0,200

Lait : 2,8 -

Deux faits sont à considérer, dit MARCQ :

" a) $\mathrm{Y}$ a-t-il corrélation chez un animal, à un moment donné, 
entre le taux butyreux sanguin et la richesse du lait en matière grasse ?

(b) Des deux sujets observés par nous, et en principe celui-là qui fournit un sang à richesse moyenne supérieure en matière grasse pendant une période d'observation encore à déterminer, fournit-il un lait à richesse moyenne supérieure durant toute la période de lactation.

« Il est nécessaire d'être en possession de nombreux résultats pour apprécier comme il convient la corrélation. Mais elle n'a pas pour nous une importance primordiale, et nous n'attendons même pas la solution du problème de ce côté.

«Il nous suffirait que la moyenne obtenue à la suite d'un certain nombre de prélèvements nous permette d'opérer un elassement des individus. Si la même observation se vérifie chez le mâle, on saisit immédiatement l'importance pratique de cette donnée pour le choix des jeunes. $)$

J. MarcQ compte reprendre ses expériences suivant un plan qu'il a adopté avec son collègue $\mathbf{M}$. A. LERox, de l'Institut agronomique de Paris. (1)

Plan d'expériences. - Nous pouvons done dire que la recherche de la vérification de l'hypothèse formulée par l'un de nous est poursuivie de différents côtés, et il est à souhaiter que les travaux s'accumulent sur cette question.

Nos expériences ont porté sur quelques vaches qui ont été mises à notre disposition par des personnes complaisantes que nous tenons à remercier ici (2).

Dès l'instant où nous voulions nous assurer s'il y avait un parallélisme entre la richesse du sang en acides gras et le taux butyreux du lait, il paraissait indispensable de comparer les échantillons prélevés, sinon exactement au même moment, car cela est impossible, mais à des moments presque voisins.

Il est évident, et c'est là une chose de première importance, que l'analyse d'un prélèvement sanguin qui ne demande que quelques secondes ne peut traduire qu'un des multiples aspects, - aspects sans doute voisins, mais tout de même différents, - que ce liquide peut présenter au cours de la journée, voire même au cours d'une partie de la journée ou même d'une heure.

Que pouvons-nous placer en face d'un échantillon dont la variabilité n'est pas niable, - bien que les limites entre lesquelles elle joue ne soient pas fort éloignées l'une de l'autre, - pour un animal donné, dans des circonstances bien déterminées ? Un prélèvement sur le

(1) Nous publierons bientôt un travail de ces deux auteurs sur la question. (R).

(2) Notre gratitude va tout particulièrement à M. Carrêt et à MHe Savoye. Qu'ils veuillent bien recevoir ici l'expression de nos remercîments les plus vifs. - CH $P$. 
contenu de la mamelle qui aura demandé plusieurs heures pour se collecter.

Or, les prélèvements successifs de lait peuvent présenter des taux butyreux dissemblables. Trouverons-nous la raison des écarts de ces derniers dans des différences, - sinon du même ordre de grandeur, mais de même sens, - dans la richesse du sang en acides gras ? Il faut confronter de nombreux chiffres pour en juger, mais a priori on peut penser que plus nous multiplierons les prises sanguines, et parallèlement les mulsions, mieux il nous sera possible de voir si la richesse du sang en acides gras et le taux butyreux du lait correspondant peuvent marcher de pair, quoique sur des paliers différents.

$\mathrm{Au}$ surplus, nous entrerons dans le détail des prises sanguines et des prises de lait dans les expériences qui seront relatées plus loin.

La technique. - Le dosage des acides gras dans le sang s'accompagne nécessairement de celui de l'insaponifiable. L'un et l'autre ont été faits par le procédé de Kumagawa, modifié par LAMELAND et par Terroine et ses élèves.

Ils ont été effectués sur le plasma de la veine jugulaire. On introduit une aiguille dans la veine et l'on reçoit le sang dans un flacon de $100 \mathrm{~cm}^{3}$ contenant du fluorure de sodium ou du citrate de sodium en poudre pour retarder la coagulation. L'avantage d'employer l'un ou l'autre de ces deux sels en poudre, e'est de ne pas entraîner de modifications de volume, et par suite, pas de corrections dans les résultats.

On centrifuge immédiatement le sang, et $20 \mathrm{~cm}^{3}$ du plasma sont versés goutte à goutte dans un becher contenant environ $100 \mathrm{~cm}^{3}$ d'alcool à $95^{\circ}$. On abandonne jusqu'au lendemain, ou même plus. On filtre sur une cartouche qui servira à l'extraction et on recueille le filtrat dans le ballon de l'appareil de KUMAGAwa. On ajoutę encore un peu d'alcool à $95^{\circ}$ et l'on fait une extraction pendant huit heures.

Lorsque ceci est terminé, on poursuit les opérations en utilisant la méthode décrite par P. BELIN (1) a vec quelques modifications. On saponifie avec de la potasse à $30 \%$. On pèse les acides gras et l'insa ponifiable après les avoir séchés dans le vide pendant 6 heures.

Lorsqu'on effectuait les dosages sur le sang des vaches en état de lactation, on faisait en même temps le dosage de la matière grasse dans le lait en utilisant la méthode de MEILLÈre qui ressemble à celle de Röse-Gotrtiteb. Mais, quelquefois, on a procédé à ces dosages en utilisant, pour le lait comme pour le sang, le procédé de KuMAGAWA décrit précédemment.

Tous les dosages ont été effectués en double, qu'il s'agisse du sang

(1) Betin. - Bull. Soc. Ohim. Biot., 1926, 8, 1081. 
ou du lait, et c'est la moyenne des résultats que l'on trouve exprimée dans les tableaux.

Résultats expérimentaux. - Première expérience. - On s'est d'abord adressé à une vache "sèche ", non pleine, qui dans les lactations antérieures avait été une mauvaise laitière.

Pendant la période expérimentale, elle a mangé chaque jour : $12 \mathrm{~kg}$. de luzerne, $2 \mathrm{~kg}$. de son et $1 \mathrm{~kg}$. d'orge. Cette quantité de nourriture lui était distribuée en deux fois, le matin et le soir.

On a pris, sur cette vache, 14 échantillons de sang pendant une póriode de 24 jours, et les résultats sont exprimés dans le tableau I.

En examinant ce tableau, nous constatons que, parmi les échantillons, 10 ont été pris pandant 6 jours consécutifs à des heures différentes.

Nous voulions nous assurer, en effet, si chez cette vache "sèche", il y avait des variations notables au cours de la journée. Il ressort nettement des résultats du tableau I que les acides gras et l'insa-, ponifiable ne varient pas beaucoup d'un jour à l'autre, ni avec les heures d'une même journée. En effet, on peut y comparer :

$1^{\circ}$ Deux dosages faits, l'un, le matin, et l'autre, le soir d'une même journée (ler février) ;

$2^{\circ}$ Deux dosages faits dans la même matinée, $10 \mathrm{~h}$. et $11 \mathrm{~h} .30$ (2 février).

Dans ces différents cas, les résultats obtenus sont presque identiques : la variation maximum entre les chiffres relatifs aux acides gras est de $27 \%$; mais, dans la plupart des essais, la variation est inférieure à $10 \%$. Une constatation du même ordre de grandeur peut être faite sur l'insaponifiable.

L'ésart maximum par rapport à la valeur moyenne est de 15,3\% pour les acides gras et de $14,2 \%$ pour l'insaponifiable. Les chiffres montrent donc ainsi une constance à peu près comparable à celle que Terrorne a trouvée dans le sang d'un chien à jeun.

D'autre part, Terroine a montré qu'au cours de l'absorption de la graisse, il y avait une augmentation du taux de la teneur en acides gras et en cholestérine dans le sang (insaponifiable).

Pourquoi nos résultats sont-ils si constants bien que la vache ne soit pas à jeun? - Pour se l'expliquer, il faut se rappeler que la nourriture de la vache ne contient pas beaucoup de graisse normalement. Une détermination de la matière grasse des aliments fournis à cet animal indique une faible teneur de $2,6 \%$ de graisse ; $6,6 \%$ seulement des calories sont fournies par cette dernière.

Nous pouvions pen ier également que la digestion et l'absorption sont des phénomènes à peu près continus chez les ruminants, en raison de la disposition de leurs gros réservoirs stomacaux qui expli- 
quent la rumination. On ne peut donc pas dire qu'il y ait quelque anomalie dans cette constance relative à la matière grasse du sang. chez une vache qui n'est pas à jeun.

Les chiffres du tableau I mettent en évidence un parallélisme entre les acides gras et l'insaponifiable, mais le rapport : insaponifiable acides gras présente des oscillations plus étendues que celles. trouvées par TeRRorne pour le rapport : $\frac{\text { cholestérine }}{\text { acides gras. }}$

TABLEAU I. ACIDES GRAS ET INSAPONIFIABLE

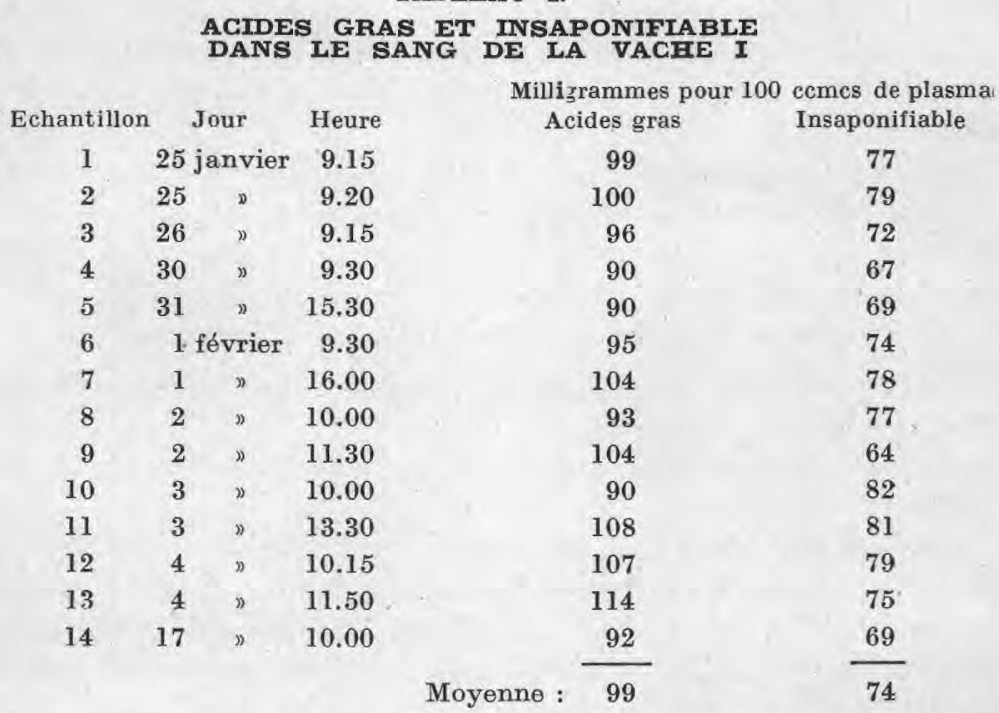

Deuxième expérience. - Toute une série de recherches ont été entreprises sur une vache dont l'accouchement était proche. Le sang fut prélevé pendant 14 jours jusqu'à la parturition, puis, a près cet événement, pendant 6 jours. Douze jours après, c'est-à-dire le $18^{\mathrm{e}}$ jour après la délivrance, 11 échantillons de sang furent pris en 5 jours consécutifs. Au cours de cette période, le dosage de la matière grasse du lait fut fait après les trois traites quotidiennes. Après une autre période de 11 jours, 10 échantillons de sang furent recueillis en 6 jours, et les dosages de la matière grasse du lait furent effectués simultanément.

Les résultats de tous ces essais sont rassemblés dans le tableau II.

Pour en faciliter la discussion, nous les avons réunis en $\mathbf{5}$ groupes correspondant chacun à une période.

Le premier groupe concerne les 14 jours avant la parturition. Pendant cette période, la vache a mangé chaque jour : $12 \mathrm{~kg}$. de luzerne, $2 \mathrm{~kg}$. de son et $1 \mathrm{~kg}$. d'orge. 
Lo deuxième groupe concerne les 6 jours après la parturition. On a augmenté graduellement la nourriture durant cette période. Le dernier jour, la vache mangeait : $15 \mathrm{~kg}$. de luzerne, $2 \mathrm{~kg}$. de son et $3 \mathrm{~kg}$. d'orge.

Le troisième groupe concerne une période dans laquelle tous les échantillons, excepté le $\mathrm{n}^{0} 52$, ont été obtenus à des heures telles que l'on puisse étudier le rapport qui existe entre la matière grasse dans le sang et la matière grasse dans le lait des traites différentes. La vache a mangé chaque jour : $15 \mathrm{~kg}$. de luzerne, $3 \mathrm{~kg}$. de son et $3 \mathrm{~kg}$. d'orge.

TABLEAU II.

\section{ACIDES GRAS ET INSAPONIFIABLE}

DANS LE SANG DE LA VAGHE II.

\begin{tabular}{|c|c|c|c|c|}
\hline \multirow[t]{8}{*}{ Période } & $\begin{array}{l}\text { Echan- } \\
\text { tillon }\end{array}$ & \multicolumn{2}{|c|}{ Jour } & Heur \\
\hline & 20 & \multicolumn{2}{|c|}{11 février } & 9.30 \\
\hline & 21 & 12 & " & 8.45 \\
\hline & 22 & 13 & » & 9.45 \\
\hline & 23 & 14 & $"$ & $8: 45$ \\
\hline & 24 & 15 & $"$ & 9.30 \\
\hline & 25 & 16 & $n$ & 10.00 \\
\hline & 26 & 17 & 》 & 10.00 \\
\hline \multirow[t]{7}{*}{1} & 27 & 18 & ” & 10.00 \\
\hline & 28 & 19 & " & 9.30 \\
\hline & 29 & 20 & $n$ & 10.00 \\
\hline & 30 & 21 & ” & 10.00 \\
\hline & 31 & 22 & $n$ & 11.00 \\
\hline & 32 & 23 & $"$ & 16.45 \\
\hline & 33 & 24 & $"$ & 10.0 \\
\hline
\end{tabular}

Moyenne :

Milligrammes pour $100 \mathrm{ccmes}$ de plasma Acide gras Insap onifiable

$\begin{array}{rr}129 & 86 \\ 132 & 83 \\ 151 & 87 \\ 164 & 93 \\ 141 & 80 \\ 146 & 86 \\ 151 & 93 \\ 153 & 90 \\ 153 & 85 \\ 177 & 123 \\ 153 & 90 \\ 154 & 90 \\ 139 & 85 \\ 151 & 88 \\ 150 & 90\end{array}$

\begin{tabular}{rrrrr} 
& 34 & 25 & " & 14.30 \\
& 35 & 26 & " & 9.00 \\
2 & 36 & 27 & " & 10.00 \\
& 37 & 28 & " & 10.15 \\
& 38 & 29 & " & 9.45 \\
& 39 & \multicolumn{2}{r}{1 mars } & 10.00
\end{tabular}

138

145

141

160 86

83

\begin{tabular}{|c|c|c|c|c|}
\hline \multicolumn{2}{|c|}{40} & 13 & $n$ & 10.00 \\
\hline & 41 & 13 & " & 14.30 \\
\hline & 42 & 14 & $n$ & 10.00 \\
\hline & 43 & 14 & ") & 14.30 \\
\hline & 44 & 15 & " & 9.45 \\
\hline & 45 & 15 & ” & 11.00 \\
\hline & 46 & 16 & » & 13.30 \\
\hline & 47 & 16 & ” & 17.00 \\
\hline & 48 & 17 & ” & 7.00 \\
\hline & 49 & 17 & $n$ & 11.00 \\
\hline
\end{tabular}




\section{TABLEAU II (suite)}

\begin{tabular}{|c|c|c|c|c|c|c|}
\hline \multirow[t]{4}{*}{ Période } & $\begin{array}{l}\text { Echan- } \\
\text { tillon }\end{array}$ & \multicolumn{2}{|c|}{ Jour } & Heure & \multicolumn{2}{|c|}{ Milligrammes pour $100 \mathrm{ccms}$ de plasma } \\
\hline & 50 & 17 & 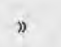 & 17.00 & 217 & 174 \\
\hline & 52 & 21 & ). & 10.50 & 194 & 156 \\
\hline & & & & Moyenne : & 195 & 147 \\
\hline \multirow{6}{*}{4} & 53 & 28 & $n$ & 16.30 & 193 & 153 \\
\hline & 54 & 29 & $\rightarrow$ & 9.30 & 166 & 145 \\
\hline & 55 & 29 & $n$ & 15.30 & 184 & 156 \\
\hline & 56 & 30 & ” & 9.15 & 209 & 154 \\
\hline & 57 & 30 & ") & 14.30 & 178 & 147 \\
\hline & & & & Moyenne : & 186 & 151 \\
\hline \multirow{6}{*}{5} & 58 & 31 & " & 10.30 & 180 & 158 \\
\hline & 59 & 31 & 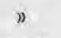 & 17.30 & 193 & 150 \\
\hline & 90 & & vril & 8.30 & 191 & 159 \\
\hline & 91 & 2 & $n$ & 10.30 & 168 & 127 \\
\hline & 92 & 2 & $n$ & 15.30 & 194 & 149 \\
\hline & & & . & Moyenne: & 185 & 148 \\
\hline
\end{tabular}

Les $4^{\mathrm{e}}$ et $5^{\mathrm{e}}$ groupes renferment les résultats d'une expérience relative à l'influence que peut exercer une augmentation de la matière grasse dans la nourriture. Pendant la quatrième période, la vache a été nourrie de la même façon qu'au cours de la troisième période, mais dans la cinquième on a ajouté chaque jour $2 \mathrm{~kg}$. de tourteau d'arachide, augmentant ainsi d'environ $40 \%$ la matière grasse de la nourriture.

Les résultats de la période 1 du tableau II mettent en évidence, comme ceux du tableau I, que les acides gras et l'insaponifiable ne varient pas beaucoup d'un jour à l'autre. La variation entre les chiffres les plus élevés et les chiffres les plus bas est plus grande que celle trouvée dans le tableau I à cause des valeurs élevées c'e l'échantillon 29. Tous les chiffres de ce premier groupe sont beaucoup plus élevés que ceux du tableau $I$. Ceci peut très bien représenter une variation individuelle, puisque la teneur du sang en lipoïdes est bien différente chez les divers sujets normaux d'une même espèce (chien).

Il est cependant possible que la matière grasse dans le sang augmente quand la vache est près d'accoucher; nous allons nous en assurer.

Hermann et Neumann (loc. cit.) ont trouvé, ainsi que nous. l'avons indiqué, une augmentation de $30 \%$ de la matière grasse dans. le sang vers la fin de la grossesse chez la femme.

Cette question de la mobilisation des graisses à la fin du part mérite d'être étudiée sur un grand nombre de sujets.

Les chiffres relatifs aux 6 jours après la parturition sont un peu plus variables que ceux qui sont obtenus avant.. Ils sont plus élevés à la fin de cette période qu'au commencement, mais la moyenne n'est 
pas sensiblement plus grande que celle de la période I. Il n'apparaît done pas qu'il y ait une augmentation immédiatement après l'accouchement. Cette augmentation est nettement plus marquée dans la période $3 ; 12$ jours se sont écoulés depuis la fin de la période 1 . La vache produit maintenant à peu près $18 \mathrm{~kg}$. de lait par jour, (Voir le tableau III) tandis que, pendant la période 1 , elle a sécrété principalement du colostrum. Tant les acides gras que l'insaponifiable du sang ont un taux plus élevé dans la troisième période. Cette augmentation est-elle le résultat d'une mobilisation plus marquée de la matière grasse empruntée aux réserves en vue de la sécrétion lactée ou dérive-t-elle d'une augmentation de la graisse ce la nourriture ? - C'est une question que nous nous sommes posé et que nous avons cherché à résoudre par la suite.

Durant les $4^{\mathrm{e}}$ et $5^{\mathrm{e}}$ périodes, la matière grasse de l'alimentation a été augmentée de $40 \%$. Or, les chiffres relevés à ce moment mettent en évidence que cette augmentation n'a pas abouti à un accroissement des acides gras et de l'insaponifiable dans le sang. On est ainsi conduit à admettre que l'élévation des acides gras et de l'insaponifiable dans le sang durant la troisième période est le fait surtout d'une mobilisation plus marquée de la graisse de dépôts en vue de répondre à la formation de la graisse du lait par la cellule mammaire, mais cette hypothèse ne nous permet toutefois pas d'éliminer tout à fait l'action possible des graisses dérivant ássez rapidement des hydrates de carbone alimentaires.

Meigs, Blatherwiok et Cary (1) ont trouvé un taux pour le phosphore lipoïdique plus élevé dans le sang des vaches en lactation que dans celui des vaches "sèches ". C'est ainsi que, pour un animal, ils ont trouvé pour 1.000 grammes de sang $0 \mathrm{gr}$. 543 de lipoïdes totaux avant l'accouchement, et 0 gr. 783 après l'accouchement.

S'opposant à ces résultats, MANSFELD trouve que le pourcentage de la matière grasse dans le sang d'une chienne est aussi élevé avant la part et à la fin de la lactation que pendant la lactation.

Leathes et Raper, en rappelant le travail de HiLleer, Linder, LUNDSGAARD et VAN SLYKE, estiment qu'un métabolisme actif de la graisse, comme il ne peut pas manquer d'en avoir pendant la sécrétion lactée, ne signifie pas nécessairement que le sang ait un pourcentage plus élevé d'acides gras.

Dans le tableau III, nous avons mis l'un à côté de l'autre les dosages des acides gras et de l'insaponifiable pour 100 gr. de plasma, le taux butyreux du lait, la quantité de lait produite ainsi que la quantité totale de matière grasse sécrétée. On voit, d'après ce tableau, que des échantillons de sang ont été pris deux ou trois fois par jour à des heures différentes, quelquefois à peu près exactement entre

(1) Meigs, Blatherwiok et Cary. - Journ. of Biol. Ohim., 1919, t. 37, pp. 1-75. 
TABLEAU III.

RAPPORT ENTRE LA MATIËRE GRASSE DANS LE SANG ET LA MATIÉRE GRASSE DANS LE LAIT. - VAGHE II

\begin{tabular}{|c|c|c|c|}
\hline $\begin{array}{l}\text { Echan- } \\
\text { tillon }\end{array}$ & \multicolumn{2}{|c|}{ Jour } & Heure \\
\hline 120 & \multicolumn{2}{|c|}{13 mars } & 6.30 \\
\hline 40 & $"$ & $"$ & 10.00 \\
\hline 121 & $"$ & ” & 11.30 \\
\hline 41 & $»$ & ” & 14.30 \\
\hline 122 & $\eta$ & 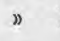 & 18.00 \\
\hline 123 & 14 & $n$ & 6.30 \\
\hline 42 & D) & $"$ & 10.00 \\
\hline 124 & 》 & $"$ & 11.30 \\
\hline 43 & ” & $n$ & 14.30 \\
\hline 125 & $"$ & " & 18.00 \\
\hline 126 & 15 & $n$ & 6.30 \\
\hline 44 & » & $"$ & 9.45 \\
\hline 45 & $”$ & $n$ & 11.00 \\
\hline 127 & ') & " & 11.30 \\
\hline 128 & ) & $n$ & 18.00 \\
\hline 129 & 16 & $"$ & 6.30 \\
\hline 130 & 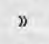 & ” & 11.30 \\
\hline 46 & 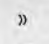 & $n$ & 13.30 \\
\hline 47 & $"$ & $n$ & 17.00 \\
\hline 131 & $"$ & " & 18.00 \\
\hline 48 & 17 & aars & 7.00 \\
\hline 132 & $"$ & $"$ & 7.30 \\
\hline 49 & 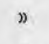 & $"$ & 11.00 \\
\hline 133 & $”$ & ” & 11.30 \\
\hline 50 & $"$ & $n$ & 17.00 \\
\hline 134 & $"$ & $”$ & 18.00 \\
\hline 135 & 28 & aars & 6.30 \\
\hline 136 & $"$ & $"$ & 11.30 \\
\hline 53 & " & v & 16.30 \\
\hline 137 & ” & $"$ & 18.00 \\
\hline 138 & 29 & $"$ & 6.30 \\
\hline 54 & $"$ & " & 9.30 \\
\hline 139 & " & " & 11.30 \\
\hline 55 & $n$ & $"$ & 15.30 \\
\hline 140 & " & ") & 18.00 \\
\hline 141 & 30 & $n$ & 6.30 \\
\hline 56 & 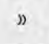 & $"$ & 9.15 \\
\hline 142 & " & $"$ & 11.30 \\
\hline 57 & " & 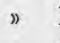 & 14.30 \\
\hline 143 & $n$ & $n$ & 18.00 \\
\hline 144 & 31 & 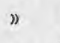 & 6.30 \\
\hline 58 & $n$ & 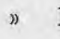 & 10.30 \\
\hline
\end{tabular}

Milligrammes pour Grammes de 100 cc plasma matière grasse Acides Insaponi- pour $100 \mathrm{cc}$ gras fiable de lait

Production en grammes

Lait Matière

192

141

190

$$
\text { 4, } 130
$$

8500

351,05

(1)

196

208

14

\section{1}

\section{9}

$\begin{array}{lll}6,390 & 3800 & 242,82\end{array}$

$\begin{array}{lll}3,850 & 4200 & 161,70 \\ 1,654 & 8500 & 140,59\end{array}$

208

\section{6}

190

145

157

$\begin{array}{lll}7,353 & 4000 & 294,12 \\ 4,246 & 4200 & 178,33 \\ 2,530 & 8500 & 215,05\end{array}$

176
195

182

198

217

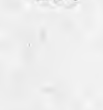

183
166
184
209
178

13

141

$\begin{array}{rll}5,206 & 4000 & 208,24 \\ 3,356 & 5000 & 167,80 \\ 3,260 & 8500 & 277,10 \\ 5,534 & 4200 & 232,43\end{array}$

134
143

$\begin{array}{rrrr}141 & 4,520 & 5500 & 248,60 \\ & 2,230 & 9500 & 211,85\end{array}$

$145 \quad 7,165 \quad 4000 \quad 286,60$

$\begin{array}{llll} & 3,980 & 5000 & 199,00 \\ & 1,427 & 9000 & 128,43 \\ 153 & 4,410 & 3200 & 141,12 \\ & & & \\ & 2,782 & 4600 & 127,97 \\ 145 & 2,248 & 8500 & 191,08 \\ & & & \\ 156 & 6,530 & 4500 & 293,85 \\ & 4,714 & 5000 & 238,70 \\ & 4,687 & 9000 & 421,83 \\ 154 & & & \\ & 6,700 & 5000 & 335,00 \\ 147 & & & \\ & 4,498 & 4500 & 202,41 \\ & 4,002 & 8500 & 340,17\end{array}$


TABLEAU III (suite).

\begin{tabular}{|c|c|c|c|}
\hline 145 & D & D & 11.30 \\
\hline 59 & " & ". & 17. \\
\hline 146 & 》 & D & 18. \\
\hline 147 & & vril & 6. \\
\hline 90 & " & $"$ & \\
\hline 148 & " & D) & 11. \\
\hline 149 & n & 》) & 18. \\
\hline 150 & 2 & 》 & 6 \\
\hline 91 & " & D & 10. \\
\hline 151 & » & D & 11. \\
\hline 9 & n & n & 15. \\
\hline 152 & 》 & 》 & 20. \\
\hline
\end{tabular}

5,653

5000

282,65

193

$191 \quad 159$

$91 \quad 159$

150

$\begin{array}{lll}3,384 & 8000 & 270,72\end{array}$

$5,200 \quad 5000$

260,00

$3,122 \quad 4500 \quad 140,49$

$4,380 \quad 9000 \quad 394,20$

168

127

194

5,532

4500

248,94

149

154,08

deux traites consécutives, d'autres fois, un peu avant la traite, d'autres fois enfin, un peu après la traite. Nous voulions en somme nous placer dans les circonstances les plus variées pour juger au mieux la relation que nous supposions devoir exister entre les acides gras du sang et la matière grasse du lait.

Les chiffres donnant le pourcentage de la matière grasse dans le lait témoignent des oscillations considérables d'une traite à une autre. C'est un fait courant de la physiologie de la lactation que nous avons rappelé au début de ce mémoire et sur lequel l'un de nous (Ch. PoRCHER) a insisté avec tant de raisons dans son travail : "Le Procès de la Matière grasse. "

Le taux butyreux est en général le plus élevé à la traite de midi, le plus bas, à la traite du matin. Un exemple choisi parmi les plus frappants se rapporte à la journée du 14 mars. On voit que, ce jour, le lait de midi est 4,5 fois plus riche en matière grasse que le lait du matin.

Les chiffres de la dernière colonne du tableau III montrent également que la production de la graisse varie beaucoup d'une traite ̀े une autre, fréquemment du simple au double. Ces grandes oscillations de la richesse du lait en matière grasse contrastent fortement avec les chiffres à peu près constants trouvés pour les acides gras dans le sang, puisque les écarts entre les plus élevés et les plus bas ne dépassent que rarement $20 \%$.

L'étude attentive de tous les résultats rassemblés ici n'indiquent pas que l'augmentation du pourcentage de la matière grasse dans le lait soit le résultat d'une augmentation d'un pourcentage du même ordre de grandeur des acides gras dans le sang pendant la période examinée de la sécrétion lactée. Rappelons d'ailleurs le fait que les laits séparés des quatre trayons de la même glande ont des richesses variables en matière grasse (Ch. PoRCHER, loc. cit., p. 92-93). Ce fait, important au point de vue physiologique et que nous avons évoqué 
à nouveau au début de ce travail, semble bien indiquer que la variation de la graisse dans le lait, s'agirait-il cette fois de traites différentes, ne peut pas fatalement correspondre à des variations de même sens des acides gras dans le sang.

Troisième expérience - Il s'agit d'une vache qui est au troisième mois de sa lactation. Les résultats des analyses sont rassemblés dans le tableau IV.

Dans le premier groupe, 9 échantillons de sang ont été pris en 4 jours consécutifs. Durant cette période, la vache a mangé par jour : $15 \mathrm{~kg}$. de luzerne, $4 \mathrm{~kg}$. de son et $4 \mathrm{~kg}$. d'orge. Pendant la deuxième, la vache a mangé la même nourriture, mais cette période fut immédiatement suivie par la troisième dans laquelle on a ajouté $2 \mathrm{~kg}$. de tourteau d'arachide par jour augmentant la graisse dans l'alimentation de $40 \%$.

TABLEAU IV.

ACIDES GRAS ET INSAPONIFIABLE

DANS LE SANG DE LA VACHE III

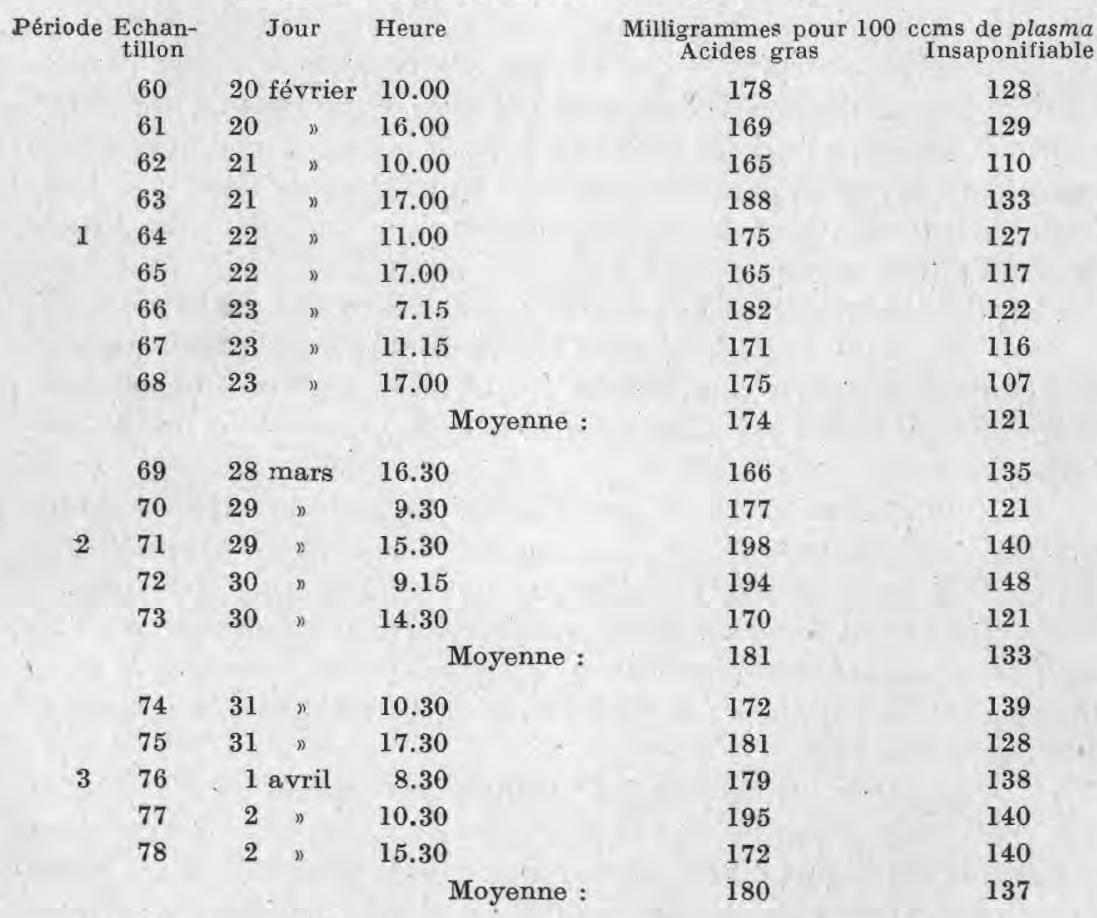

Les chiffres du tableau IV concordent bien avec ceux du tableau II, en ce sens que les acides gras et l'insaponifiable ne varient pas beaucoup d'un jour à l'autre ou à des heures différentes d'une même journée. L'augmentation de la graisse dans la nourriture au cours 
de la période 3 ne produit pas une augmentation de lipoïdes dans le sang. Nous pouvons également dire, pour cet animal, que les valeurs trouvées pour les acides gras et l'insa ponifiable sont plus faibles que celles que nous avons obtenues avec la vache précédente (tableau II, périodes 3,4 et 5), mais le même parallélisme existe.

On a prosédé également au dosage de la matière grasse dans le lait de vache III à chaque traite pour étudier le rapport qui peut exister entre la matière grasse dans le sang et celle du lait. Les résultats sont groupés dans le tableau V.

TABLEAU V.

\section{RAPPORT ENTRE LA MATIËRE GRASSE DANS LE SANG} ET LA MATIERE GRASSE DANS LE LAIT. - VAGHE III

\begin{tabular}{|c|c|c|c|c|c|c|c|c|c|c|c|}
\hline \multirow{2}{*}{\multicolumn{2}{|c|}{$\begin{array}{l}\text { Echan- } \\
\text { tillon }\end{array}$}} & \multirow[t]{2}{*}{ Jour } & \multirow{2}{*}{ Heure } & \multicolumn{2}{|c|}{$\begin{array}{l}\text { Milligrs. pour } \\
100 \mathrm{~cm}^{3} \mathrm{de} \\
\text { plasma }\end{array}$} & \multicolumn{3}{|c|}{$\begin{array}{l}\text { Milligis. pour } 100 \mathrm{~cm}^{3} \\
\text { Méthode de Kumagawa }\end{array}$} & \multirow{2}{*}{$\begin{array}{l}\text { de lait } \\
\text { Méthode } \\
\text { Meillière } \\
\text { Matière } \\
\text { grasse }\end{array}$} & \multicolumn{2}{|c|}{$\begin{array}{l}\text { Production en } \\
\text { grammes }\end{array}$} \\
\hline & & & & $\begin{array}{l}\text { Acides } \\
\text { gras }\end{array}$ & $\begin{array}{l}\text { Insapo- } \\
\text { nifiable }\end{array}$ & $\begin{array}{l}\text { Acides } \\
\text { gras }\end{array}$ & $\begin{array}{l}\text { Insapo- } \\
\text { nifiable }\end{array}$ & $\begin{array}{l}\text { Matière } \\
\text { grasse }\end{array}$ & & Lait & $\begin{array}{l}\text { Matière } \\
\text { grasse }\end{array}$ \\
\hline 1002 & $20 \mathrm{ft}$ & évrier & 6.30 & & & 2386 & 80 & 2576 & 2680 & 6300 & 168,84 \\
\hline 60 , & $"$ & $"$ & 10.00 & 178 & 128 & & & & & & \\
\hline 101 & $n$ & $n$ & 11.30 & & & 3704 & 130 & 4004 & 4150 & 2200 & 91,30 \\
\hline 61 & " & " & 16.00 & 169 & 129 & & & & & & \\
\hline 102 & $"$ & $"$ & 18.00 & & & 3098 & 110 & 3351 & 3410 & 2900 & 98,89 \\
\hline 1032 & & $n$ & 6.30 & & & 2600 & 76 & 2796 & 2915 & 6800 & 198,22 \\
\hline 62 & $"$ & $"$ & 10.00 & 165 & 110 & & & & & & \\
\hline 104 & $"$ & " & 11.30 & & & 3712 & 114 & 3997 & 4370 & 2400 & 104,88 \\
\hline 63 & $n$ & " & 17.00 & 188 & 133 & & & & & & \\
\hline 105 & 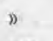 & $"$ & 18.00 & & & 3088 & 80 & 3310 & 3715 & 3000 & 111,45 \\
\hline 106 & 22 & " & 6.30 & & & 2460 & 84 & 2657 & 2800 & 6700 & 187,60 \\
\hline 64 & $n$ & $"$ & 11.00 & 175 & 127 & & & & & & \\
\hline 107 & " & $n$ & 11.30 & & & 5392 & 130 & 5770 & 6020 & 2800 & 168,56 \\
\hline 65 & $n$ & $n$ & 17.00 & 165 & 117 & & & & & & \\
\hline 108 & $n$ & $n$ & 18.00 & & & 3320 & 112 & 3585 & 3990 & 2700 & 107,73 \\
\hline 1092 & 23 & $"$ & 6.30 & & & 2582 & 82 & 2783 & 2806 & 6500 & 182,39 \\
\hline 66 & $"$ & $n$ & 7.15 & 182 & 122 & & & & & & \\
\hline 67 & $"$ & " & 11.15 & 171 & 116 & & & 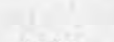 & & & \\
\hline 110 & $"$ & " & 11.30 & & & 4376 & 124 & 4601 & 4886 & 2700 & 131,92 \\
\hline 68 & " & $"$ & 17.00 & 175 & 107 & & & & & & \\
\hline 111 & $"$ & $n$ & 18.00 & & & 3202 & 102 & 3451 & 3718 & 2600 & 96,67 \\
\hline
\end{tabular}

Nous avons utilisé la méthode de MeILL̀̀Re, et, en même temps, la méthode de KUMagawa qui nous a permis de séparer l'insaponifiable des acides gras proprement dits.

Les chiffres inscrits dans la colonne "matière grasse " sous "méthode de KUMAGawa " ont été calculés en multipliant les valeurs obtenues pour les acides gras par 1,046 afin de les transformer en glycérides, et en ajoutant ensuite les valeurs trouvées pour l'insaponifiable. 
Quand on fait la comparaison entre les deux valeurs trouvées pour la matière grasse par les deux méthodes, on voit que celles qui sont déterminées par la méthode de KUMAGAwa sont toujours un peu plus basses, eomme on pouvait le prévoir. Les différences entre les deux colonnes sont cependant assez constantes et ne dépassent jamais $10 \%$.

Les chiffres montrent que l'insaponitiable ne constitue qu'un pourcentage assez faible de la matière grasse totale du lait, tandis que, dans le sang, l'insaponifiable correspond à près de $40 \%$ de la matière grasse totale.

Nous n'avons pris qu'un échantillon de $5 \mathrm{~cm}^{3}$ pour doser l'insaponifiable du lait, et peut-être $\epsilon$ st-ce là une prise trop faible en vue d'un dosage précis. Mais nous ne pensons pas que les erreurs expérimentales puissent tout de même modifier notre façon de voir.

Nous notons également, si nous rapprochons les chiffres du tableau V de ceux du tableau III, que si les variations de la matière grasse pour la vache III sont très grandes d'une traite à l'autre, il n'y a rien de semblable pour les variations des acides gras dans les prises sanguines correspondantes. Encore une fois, pour cet animal, comme pour les précédents, les taux des acides gras et de l'insaponifiable dans le sang n'éprouvent pas beaucoup d'oscillations aux heures différentes du même jour, malgré les grandes amplitudes du taux butyreux que nous constatons dans le lait.

Les conclusions que nous avons tirées de l'examen du tableau III sont done renforcées par celles que nous pouvons extraire du tableau V.

Quatrième expérience. - Il n'est pas sans intérêt de comparer le quantum des acides gras et de l'insaponifiable dans le sang de la veine jugulaire et le sang de la veine mammaire, afin de déterminer s'il était possible de trouver quelque abaissement de ces lipoïdes lorsque le sang a passé par la glande mammaire.

Les deux échantillons des deux sangs ont été pris à peu près simultanément.

Le tableau VI rassemble les résultats trouvés.

TABLEAU VI

RAPPORT DES AGIDES GRAS ET DE L'INSAPONIFIABLE DANS LA VEINE JUGULAIRE ET LA VEINE IMAIVIV:AIRE

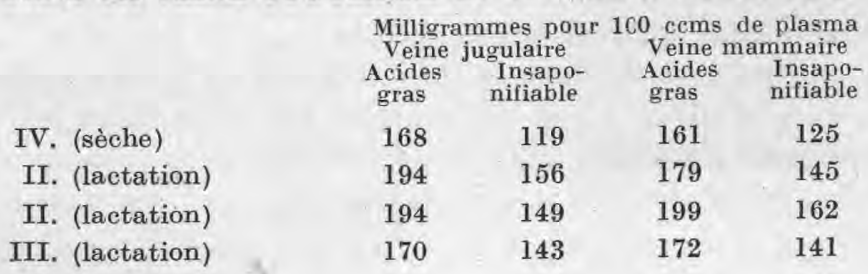


Les deux premiers chiffres se rapportent à une génisse "sèche ", mais a priori on pouvait supposer ne pas trouver de différences sensibles dans les dosages du sang de la veine jugulaire et ceux du sang de la veine mammaire. Au eontraire, pour les animaux en lactation, on devait admettre une différence certaine. Or, les résultats trouvés pour les trois animaux en lactation ne concordent pas du tout. Alors que, pour l'animal II, cette différence n'est pas douteuse et est nette pour la première prise, elle ne l'est pas pour la seconde et les résultats observés sont de sens contraires. Pour l'animal III, il en est de même:

Meigs, Blatherwick et CaRy (loc. cit.) ont montré par le calcul, en y joignant certains raisonnements en vue de corriger les chiffres obtenus, que l'abaissement de la matière grasse dans le sang en passant par la glande mammaire est seulement de 9 milligrammes pour $100 \mathrm{em}^{3}$ de sang, abaissement vraiment trop faible à mesurer avec exactitude.

Des recherches nombreuses et prolongées doivent être reprises dans cette direction.

\section{Conclusions.}

Si le nombre des animaux sur lesquels nous avons pu travailler est restreint, du moins avons-nous fait sur eux de multiples prélèvements dont on peut dire, semble-t-il, que le dépouillement nous permet de tirer quelques conclusions intéressantes des résultats qui en découlent.

Bien loin de nous la prétention de les croire définitives, car l'intérêt de la question soulevée ici est si grand que de multiples investigations orientées dans le même sens que celles qui sont décrites ici sont nécessaires avant de conclure quelque peu solidement. Peuton dire que le taux des acides gras dans le sang s'élève quand l'accouchement approche? C'est assez vraisemblable, mais nous n'avons pas ássez de chiffres pour l'avancer très nettement. II ne serait pas surprenant qu'il y eât pour la matière grasse comme pour le glucose une mobilisation en vue de la sécrétion qui va s'établir. Pour le g]ucose, Ch. Poncher a montré qu'il y a une véritable poussée de la glycémie au moment du part. Cette mobilisation est peut-être moins rapide pour la graisse que pour le glucose. Toutefois, il est bien de nous rappeler que l'observation relevée par HTLLER, LINDER, LUNDSGAARD et VAN SLYKE, au cours de laquelle ces auteurs estiment qu'un métabolisme très actif de la graisse, comme il doit en être pendant la sécrétion lactée, ne signifie pas nécessairement un pourcentage plus élevé dans le sang; ils y voient comme la preuve indirecte que la graisse doit se mobiliser assez vite.

Il est un point qui n'a pas été abordé dans notre travail, faute de temps et surtout faute de matériel. Il eut été, en effet, fort intéressant 
de comparer les constantes de la graisse du sang à celles de la graisse du lait. Comme le sang n'est pas riche en matière grasse, il aurait fallu opérer sur une quantité considérable de plasma, afin d'obtenir des échantillons copieux permettant toutes les déterminations analytiques.

Les résultats relevés dans ce travail nous montrent que le rapport du quantum des acides gras du sang à celui de l'insaponifiable est faible, oscillant autour de 1,25 à 1,40. Au contraire, dans le lait, le même rapport est considérable, car l'on peut dire qu'il y a réellement fort peu d'insa ponifiable par rapport aux acides gras dans cette sécrétion.

Nous avons déjà eu l'occasion d'insister, tellement il nous semble que cette observation avait d'importance, sur la nécessité qu'il y aurait de déterminer aussi exactement que possible la quantité de sang qui traverse une mamelle pendant 24 heures, à l'état de repos et en plein travail fonetionnel.

Doser la matière grasse dans le lait, c'est effectuer une analyse sur un liquide à l'état de repos, liquide dans lequel se sont concentrés en quelque sorte, avec l'aide du temps, les éléments que l'on veut y déterminer. Effectuer cette fois un dosage des éléments correspondants dans le sang, c'est analyser un liquide en circulation qui se renouvelle à ehaque instant, liquide dont le taux des acides gras est toujours faible. On aurait tort de parler de concentration des acides gras dans le sang, car cette expression serait sans valeur.

II suffit peut-être d'une très légère variation dans le taux d'un élément du sang, ici les acides gras, pour que lui corresponde cette fois une véritable concentration de cet élément en un autre point, mettons l'intérieur de la glande mammaire.

Les premiers calculs, très approximatifs, de la quantité de sang qui traverse la mamelle pendant 24 heures fixent à plusieurs milliers de litres cette dernière. Il suffit done qu'il soit fait sur le sang un prélèvement très faible d'une quantité d'un principe donné, prélèvement auquel, analytiquement parlant, nous n'attachons pas toujours l'importance qu'on devrait cependant lui reconnaître, pour que se concentre dans le lait une pius grande quantité de matière grasse.

D'après les recherches qui ont été poursuivies pendant longtemps sur deux des vaches dont nous avons pu disposer, il y a nettement et continûment une différence de richesse moyenne en acides gras du sang de ces deux animaux. On pourrait estimer que le mot nettement est exagéré, mais à la réflexion, c'est moins la grandeur de la différence qui importe que sa constance, et e'est ce qui donne à ce fait toute sa valeur.

Comment alors le concilier avec l'observation que nous avons faite plus haut, lorsqu'au sujet des variations faibles de la graisse dans le sang nous signalions l'absence de parallélisme marqué entre 
la graisse du sang et celle du lait ? Sur quoi la notion chimique du sang butyreux doit-elle alors s'appuyer?

Le taux de la matière grasse du lait chez un animal donné, quand on considère la suite de ses traites, est vraiment une variable, mais ce qui n'est plus une variable, ce qui se retrouve au cours des diverses lactations d'un même animal, et avec, cette fois, l'allure d'une constante, c'est le taux butyreux moyen de toute sa lactation. De nombreux faits nous montrent qu'un animal qui est bon butyrogène le reste pendant toute sa vie. Si aucun incident pathologique ne vient se mettre au travers de l'une ou de l'autre de ses lactations, nous retrouvons chez toutes un taux butyreux moyen qui caractérise bien l'animal au point de vue beurrier. Ce taux moyen a son image sanguine dans cette constance, - que nous retrouvons sans qu'elle ait de grands écarts, - du taux des acides gras du plasma sanguin, et de deux animaux, le meilleur beurrier est celui qui possède la plus grande richesse en acides gras par litre de plasma sanguin.

En conséquence, nous saisissons maintenant tout l'intérêt de l'analyse du sang. Le parallélisme que nous voulions établir entre les prises de sang et les prises de lait, faites au même moment pour ainsi dire, nous échappe en raison de l'irrégularité du métabolisme de la matière grasse, mais ce qui ne nous échappe plus, ce sont ces deux données que, cette fois, on peut mettre en parallèle : le taux en acides gras du sang et le taux butyreux moyen de toute la lactation. Ceci justifie done bien l'analyse du sang, car, et notre travail le montre, les chiffres que nous avons obtenus n'ont pas du tout ces très grandes oscillations qui nous frappent tant dans le taux butyreux des laits correspondants.

En fait, et nous n'avons qu'à nous reporter à nos tableaux, une seule prise de sang ne suffirait pas pour fixer la qualité butyrogène laitière d'un animal, mais du moins pourrions-nous nous contenter d'un nombre assez restreint, peut-être pas plus de cinq ou dix prises, réparties sur plusieurs semaines ou plusieurs mois, et c'est leur moyenne que nous aurions à rapprocher du taux butyreux moyen de la lactation entière. C'est à l'expérience de nots fixer sur le nombre des prises sanguines et sur le moment où elles doivent être effectuées.

Quoi qu'il en soit, voici un plan de recherches qui mérite d'être exécuté :

$1^{\circ}$ Posséder un troupeau d'animaux de qualités beurrières très différentes. Le nourrir soigneusement de façon à obtenir le meilleur rendement chez tous les sujets. Recueillir, pendant toute la durée de la lactation, la quantité du lait sécrété et doser avec soin sa matière grasse, ce qui nous permettra à la fin de la lactation, tous calculs faits, d'avoir le taux butyreux moyen vrai de la production laitière totale; 
$2^{\circ}$ Faire des prises de sang à intervalles variables; les rapprocher quelquefois, les éloigner d'autres fois. En un mot, s'arranger pour avoir un nombre important de prises. Doser dans le plasma les acides gras et l'insaponifiable. Faire la moyenne;

$3^{\circ}$ En face du taux butyreux moyen de la lactation tout entière, placer le taux moyen en acides gras par litre de plasma de l'animal et comparer.

Si la notion du "sang butyreux ", telle que l'un de nous l'a suggérée, est bonne, nous devrons avoir un parallélisme assez étroit entre les acides gras du sang et la matière grasse du lait, le meilleur beurrier étant celui dont le sang est le plus riche en acides gras.

Nous souhaitons que ces expériences soient faites. Elles coûteront de l'argent et de la peine, mais si elles sont entreprises selon le plan que nous venons de fixer, nul doute que l'étude de la physiologie de la lactation n'en recueille un grand bénéfice.

\title{
SUR LES FLORES BACTÉRIENNE ET FONGIQUE DES CAILLÉS DE LAIT
}

\author{
RÔLE DES SYMBIOSES DANS LA MATURATION \\ DU CAMEMBERT
}

par

Mademoiselle FRANÇOISE SANSONETTI

Docteur en Pharmacie

$$
\text { (Suite) }
$$

\section{ETUDE SYSTÉMATIQUE DES MOISISSURES ET DES BACTÉRIES DU CAMEMBERT}

A la Société de l'Abbaye de St-Georges d'Aunay (Calvados), nous avons fait choix en hiver de nombreux échantillons de caillé et de fromage. A l'aide d'une spatule en platine flambée, nous avons prélevé, sous la croûte, de petits fragments de caillé qui ont été déposés dans des tubes contenant du caillé stérile.

Deux Moisissures ont été isolées et étudiées : un Ö̈dium et un Penicillium. Nous a vons obtenu en culture pure différentes espèces bactériennes : des cocci de $2,8 \mu$, prenant le Gram et liquéfiant très rapidement la gélatine; des bacilles dont une espèce formée de bâtonnets de $5 \mu$ de long, aux extrémités effilées, ne prend pas le Gram. Les éléments d'une autre souche sont extrêmement courts $(0,8 \mu)$ et prennent le Gram. Nous avons étudié seulement une bactérie dont les formes d'involution sont nombreuses et qui présente un bouquet spécifique de Camembert; il en est parlé plus loin.

Sur les fromages insuffisamment salés nous avons prélevé un 